Bundesgesundheitsbl - Gesundheitsforsch Gesundheitsschutz 2005 • 48:1211-1216 DOI 10.1007/s00103-005-1159-6 Online publiziert: 22. Oktober 2005 ๑) Springer Medizin Verlag 2005

T. Ziese $\cdot H$. Neuhauser $\cdot$ Robert Koch-Institut, Berlin

\title{
Der telefonische Gesundheitssurvey 2003 als Instrument der Gesund- heitsberichterstattung des Bundes
}

gen, die sowohl unter dem Gesichtspunkt der Prävention als auch im Hinblick auf die Heterogenität der Versorgungsbedarfe und der Selbsthilfe im Gesundheitswesen zunehmend an Bedeutung gewinnen.

Die bisherigen Health Examination and Interview Surveys liefern zwar eine breite und valide Datengrundlage, sind aber aufgrund des hohen personellen, logistischen und finanziellen Aufwands nur in mehrjährigen Abständen realisierbar. Ein Gesundheitsmonitoring setzt jedoch eine kontinuierliche Beobachtung in kürzeren Zeitintervallen voraus, d. h., es muss die regelmäßige und zeitgerechte Verfügbarkeit der Daten gewährleistet sein. Nur so lassen sich aktuelle Trends aufzeigen und im Zusammenhang mit gesundheitspolitischen Interventionen bewerten [1]. Aus diesem Grund führt das Robert Koch-Institut (RKI) seit kurzem zusätzlich repräsentative telefonische Befragungen durch, mit denen die Zeitabstände zwischen den Untersuchungssurveys überbrückt werden sollen.

\section{Telefonische Gesundheitssurveys im internationalen und nationalen Kontext}

In den USA werden seit den 1950er-Jahren nationale Untersuchungssurveys (National Health Interview Survey, NHIS) als Face-to-face-Befragungen durch das $\mathrm{Na}$ tional Center for Health Statistics am Cen- ters for Disease Control and Prevention (CDC) durchgeführt. Zusätzlich hat das CDC 1984 begonnen, auch repräsentative telefonische Surveys durchzuführen, die mit deutlich weniger Kosten verbunden sind und flexibler Fragen zu aktuellen Entwicklungen aufnehmen können [2]. Diese wurden seitdem zu einem Monitoringsystem für gesundheitsrelevante Verhaltensweisen (Behavioural Risk Factor Surveillance System, BRFSS) mit monatlichen Computer Assisted Telephone Interview (CATI) in allen Bundesstaaten (insgesamt ca. 200.00o Interviews pro Jahr) weiterentwickelt. Schwerpunkt der CATI-Interviews ist das Gesundheitsverhalten (z. B. Rauchen, Alkoholkonsum, Impfungen, körperliche Aktivität, Ernährung, Verwendung von Sicherheitsgurten), es sind aber auch Fragen zu chronischen Erkrankungen und zur Inanspruchnahme medizinischer Leistungen enthalten. Die Daten werden sowohl in den einzelnen Bundesstaaten als auch auf nationaler Ebene ausgewertet und dienen der kontinuierlichen Gesundheitsberichterstattung, aber auch zur Evaluation der nationalen Gesundheitsziele „Healthy People 2010“ [3].

In den Ländern der Europäischen Union (EU) wurden nationale Gesundheitssurveys bislang nicht telefonisch durchgeführt, auch wenn eine Expertise über den Status quo und Perspektiven von Gesundheitssurveys in der EU, die im Rahmen subjektive Einstellungen, Wahrnehmungen und Verhaltensorientierungen berücksichti- 


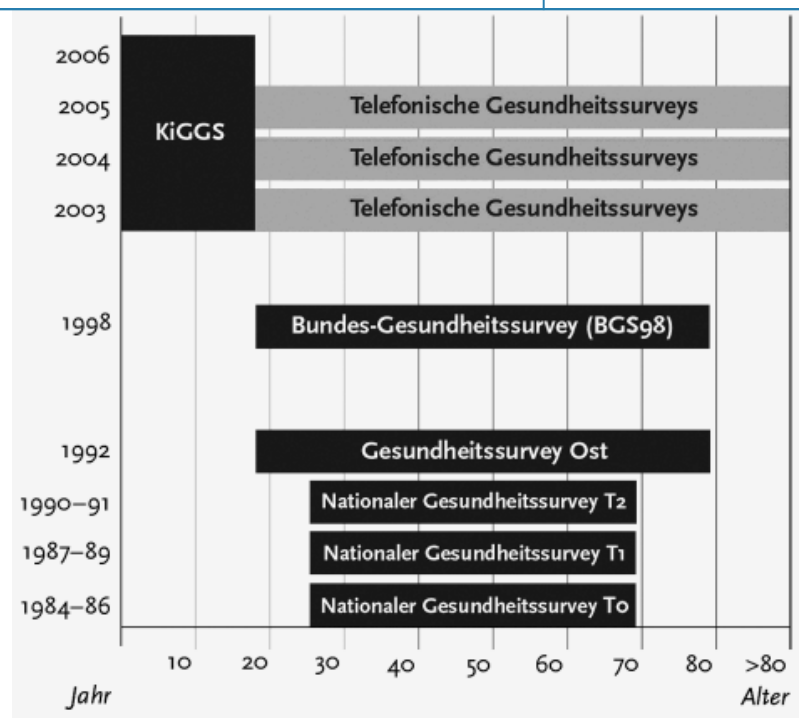

den. Dieses Cross-over-Design erlaubt es, systematische Effekte der verschiedenen Erhebungsweisen von zufälligen Effekten zu unterscheiden sowie den Einfluss der Methodik auf die Ergebnisse einzuschätzen. Damit können die durch das Erhebungsverfahren bedingten Abweichungen untereinander abgeschätzt und entsprechende Korrekturoptionen für den Aufbau von Zeitreihen und -vergleichen entwickelt werden.

\section{Eckdaten des telefonischen Gesundheitssurveys 2003}

Im Kontext des derzeit im Aufbau begriffenen Gesundheitsmonitoringsystems [1] spielen telefonische Gesundheitssurveys eine wichtige Rolle als Bindeglied zwischen den weiterhin konzeptionell vorgesehenen Untersuchungssurveys. Dem telefonischen Gesundheitssurvey 2003 (GSTelo3) kam damit die Funktion einer Pilotstudie zu. Diese Erhebung wurde mit Finanzierung durch das Bundesministerium für Gesundheit und Soziale Sicherung (BMGS) im Zeitraum von September 2002-März 2003 realisiert. Es wurden 8313 Personen repräsentativ für die deutschsprachige Wohnbevölkerung ab 18 Jahren u. a. zu Krankheiten, zu ihrem Gesundheitsverhalten und zur Inanspruchnahme von Leistungen des Gesundheitswesens befragt (vergleiche Kapitel „Themen und Instrumente“). Im Vergleich zum Bundes-Gesundheitssurvey 98 wurde die obere Altersbegrenzung von 79 Jahren aufgegeben. Unmittelbar nach Abschluss der Feldphase und der Datenaufbereitung begann die Auswertung der erhobenen Informationen, und ein deskriptiver Ergebnisbericht wurde [2] veröffentlicht.

Das Stichprobendesign bildet die volljährige, deutschsprachige Wohnbevölkerung in Privathaushalten ab, sofern sie über Festnetzanschlüsse erreichbar ist. Basis ist ein nach dem Gabler-Häder-Verfahren gezogenes und vom Zentrum für Umfragen, Methoden und Analysen (ZUMA) in Mannheim zur Verfügung gestelltes Sample von jeweils ca. 45.00o zufallsgenerierten Rufnummern aus dem Pool der über 35 Millionen privaten Telefonanschlüsse in Deutschland. Es bezieht auch die Rufnummern von Haushalten ein, die 
nicht in Telefonbüchern eingetragen sind, und ermöglicht damit eine bundesweit repräsentative Zufallsauswahl aller Privathaushalte. Die Repräsentativität auf Personenebene wird durch eine zweite Auswahlstufe erreicht: In Mehrpersonenhaushalten wird nur diejenige volljährige Person befragt, die beim ersten Kontakt als nächste Geburtstag hat (Next-birthdayMethode). Beide Teile des telefonischen Gesundheitssurveys wurden nach Alter, Geschlecht und Region gewichtet und an die Bevölkerungsstruktur des jeweiligen Bezugsjahres angepasst.

Eine ausführlichere Beschreibung der Methode und des Designs des telefonischen Gesundheitssurveys findet sich im Beitrag von M. Kohler et. al in diesem Heft. Darüber hinaus sind detaillierte Monographien über verschiedene Aspekte zur Methodik, zum Design und zur Feldphase der Telefonsurveys über die Internet-Seiten des RKI verfügbar (http://www.rki.de $\rightarrow$ Epidemiologie und Gesundheitsberichterstatttung $\rightarrow$ Datenerhebungen).

\section{Themen und Instrumente}

Die Zielsetzung der telefonischen Gesundheitssurveys entspricht denen der nationalen Untersuchungssurveys des RKI. Hierzu zählen zum einen die Bereitstellung aktueller Daten zur Verbreitung ausgewählter Erkrankungen vorwiegend mit chronischem Verlauf, zum anderen die Bereitstellung von Informationen zur Verbreitung wichtiger Risikofaktoren und zum gesundheitsbezogenen Verhalten in der bundesdeutschen Bevölkerung für Politik und Wissenschaft. Daher war ein wesentliches Kriterium bei der Entwicklung des Fragebogens des GSTelo3 seine möglichst weitgehende Übereinstimmung mit den Untersuchungssurveys des RKI, hier insbesondere mit dem Bundes-Gesundheitssurvey 1998, der wiederum in weiten Bereichen die Zeitreihen fortführt, die mit den Nationalen Gesundheitssurveys der Deutschen Herz-Kreislauf-Präventionsstudie Anfang der 1980er-Jahre begonnen wurden. Damit stehen jetzt für viele Gesundheitsaspekte Daten über einen Zeitraum von mehr als 20 Jahren zur Verfügung (• Tabelle 1).

Ein weiteres Kriterium stellte die internationale Vergleichbarkeit der erhobenen Daten dar. Zum einen wurde, soweit mög-

Bundesgesundheitsbl - Gesundheitsforsch - Gesundheitsschutz 2005 - 48:1211-1216 DOI 10.1007/s00103-005-1159-6

○) Springer Medizin Verlag 2005

T. Ziese $\cdot H$. Neuhauser

\section{Der telefonische Gesundheitssurvey 2003 als Instrument der Gesundheitsberichterstattung des Bundes}

\section{Zusammenfassung}

Im Robert Koch-Institut werden Gesundheitssurveys bereits seit den 1980er-Jahren in unregelmäßigen Abständen durchgeführt. Telefonsurveys werden international seit einiger Zeit als Ergänzung zu den Untersuchungs- und Befragungssurveys eingesetzt. Diese kostengünstige und schnelle Art der Datengewinnung ermöglicht es zum einen, Zeitreihen zur Gesundheit weiterzuführen, und zum anderen, gleichzeitig auf aktuelle gesundheitsrelevante Fragestellungen reagieren zu können, unter anderem auch deshalb bilden die telefonischen Gesundheitssurveys ei- nen wichtigen Baustein für das aufzubauende Gesundheitsmonitoringsystem in Deutschland. Von September 2002-März 2003 wurden erstmals 8313 Personen aus der deutschsprachigen Wohnbevölkerung ab 18 Jahren u. a. zu Krankheiten, zu ihrem Gesundheitsverhalten und zur Inanspruchnahme von Leistungen des Gesundheitswesens befragt.

\section{Schlüsselwörter}

Gesundheitssurveys · CATI .

Gesundheitsdaten - Gesundheitsziele .

Monitoring

\section{The German Telephone Health Survey 2003: an instrument of federal health reporting}

\section{Abstract}

The Robert Koch Institute has carried out health surveys at irregular intervals since the 1980s. Internationally telephone surveys have been used to supplement examination surveys for some time. Health interview surveys by telephone provide a timely and inexpensive possibility to build up a time series on health while being flexible enough to cover new health aspects. Health telephone surveys will also serve as a module of a coming health-monitoring system in Germany, which is currently under discus- sion. From September 2002 to March 2003, 8,313 German-speaking people randomly selected from the resident population aged 18 years and over were asked about topics such as chronic diseases, health behaviour and the extent to which they make use of health care services and others.

\section{Keywords}

Health surveys · CATI · Health data . Health targets $\cdot$ Health monitoring 
Tabelle 1

Übersicht der Themenbereiche des Bundes-Gesundheitssurveys 1998 und des Telefonischen Gesundheitssurveys 2003

\begin{tabular}{|c|c|c|}
\hline Bereich & Bundes-Gesundheitssurvey 1998 & Telefonischer Survey 2003 \\
\hline $\begin{array}{l}\text { Subjektive Gesundheitswahrnehmung } \\
\text { und gesundheitsbezogene Lebensqualität }\end{array}$ & $\begin{array}{l}\text { - Gesundheitliche Lebensqualität (SF36) } \\
\text { - Beschwerden } \\
\text { - Schmerzen } \\
\text { - Lebenszufriedenheit }\end{array}$ & $\begin{array}{l}\text { - Allgemeine subjektive Gesundheit } \\
\text { - Gesundheitsbezogene Lebensqualität (SF8) }\end{array}$ \\
\hline $\begin{array}{l}\text { Gesundheitsverhalten, Risikofaktoren, } \\
\text { Prävention }\end{array}$ & $\begin{array}{l}\text { - Erhöhtes Cholesterin } \\
\text { - Alkoholkonsum } \\
\text { - Rauchen } \\
\text { - Übergewicht } \\
\text { - Sport } \\
\text { - Körperliche Aktivität } \\
\text { - Ernährung } \\
\text { - Grippeschutzimpfung } \\
\text { - Gesundheits-Check-up } \\
\text { - Krebsfrüherkennung }\end{array}$ & $\begin{array}{l}\text { - Erhöhtes Cholesterin } \\
\text { - Alkoholkonsum } \\
\text { - Rauchen } \\
\text { - Übergewicht } \\
\text { - Sport }\end{array}$ \\
\hline Krankheitsgeschehen & $\begin{array}{l}\text { - Diabetes } \\
\text { - Atemwegserkrankungen } \\
\text { - Herz-Kreislauf-Erkrankungen } \\
\text { - Stoffwechselerkrankungen } \\
\text { - Infektionskrankheiten } \\
\text { - Muskel- und Skeletterkrankungen } \\
\text { - Psychische Erkrankungen } \\
\text { - Dermatologische Erkrankungen } \\
\text { - Allergien } \\
\text { - Unfälle und Verletzungen }\end{array}$ & $\begin{array}{l}\text { - Diabetes } \\
\text { - Atemwegserkrankungen } \\
\text { - Herz-Kreislauf-Erkrankungen } \\
\text { - Stoffwechselerkrankungen } \\
\text { - Infektionskrankheiten } \\
\text { - Muskel- und Skeletterkrankungen } \\
\text { - Psychische Erkrankungen } \\
\text { - Dermatologische Erkrankungen } \\
\text { - Allergien } \\
\text { - Unfälle und Verletzungen }\end{array}$ \\
\hline Krankheitsfolgen und Behinderung & $\begin{array}{l}\text { - Schwerbehinderung, Grad der Behinderung } \\
\text { - Minderung der Erwerbsfähigkeit, } \\
\text { Grade der Minderung der Erwerbsfähigkeit }\end{array}$ & $\begin{array}{l}\text { - Schwerbehinderung, Grad der Behinderung } \\
\text { - Minderung der Erwerbsfähigkeit, } \\
\text { Grade der Minderung der Erwerbsfähigkeit } \\
\text { - Activities of daily life (ADL) }\end{array}$ \\
\hline $\begin{array}{l}\text { Inanspruchnahme von Leistungen } \\
\text { des Gesundheitssystems }\end{array}$ & $\begin{array}{l}\text { - Hausärztliche Versorgung } \\
\text { - Inanspruchnahme Ärztegruppen } \\
\text { - Krankenhausnächte } \\
\text { - AU-Zeiten } \\
\text { - Teilnahme an Selbsthilfegruppen } \\
\text { - Inanspruchnahme Rehabilitation }\end{array}$ & $\begin{array}{l}\text { - Hausärztliche Versorgung und } \\
\text { Inanspruchnahme } \\
\text { - Krankenhausnächte } \\
\text { - Teilnahme an Selbsthilfegruppen } \\
\text { - Inanspruchnahme Rehabilitation }\end{array}$ \\
\hline $\begin{array}{l}\text { Gesundheitswissen, Informationsverhalten, } \\
\text { gesundheitliche Einstellungen }\end{array}$ & & - Informationsquellen zur Gesundheit \\
\hline Sozialer Hintergrund & $\begin{array}{l}\text { - Familienstand } \\
\text { - Lebensform } \\
\text { - Haushaltsgröße } \\
\text { - Kinderzahl } \\
\text { - Erwerbsstatus } \\
\text { - Sozialschicht } \\
\text { - Migrationshintergrund } \\
\text { - Arbeitslosigkeit } \\
\text { - Versichertenstatus }\end{array}$ & $\begin{array}{l}\text { - Familienstand } \\
\text { - Lebensform } \\
\text { - Haushaltsgröße } \\
\text { - Kinderzahl } \\
\text { - Erwerbsstatus } \\
\text { - Sozialschicht } \\
\text { - Migrationshintergrund } \\
\text { - Arbeitslosigkeit } \\
\text { - Sozialhilfebezug } \\
\text { - Versichertenstatus }\end{array}$ \\
\hline
\end{tabular}

lich, auf Instrumente zurückgegriffen, die auch im internationalen Raum in Gesundheitssurveys eingesetzt werden. Zusätzlich wurden Elemente des im Auftrag von Eurostat entwickelten Instruments „Minimum European Health Module“ eingesetzt [12]. Diese Elemente umfassen 3 Fragen (subjektive Gesundheit, gesundheitli- che Einschränkung im Alltag, chronische Krankheiten) und sind sowohl für geplante EU-weite Gesundheitssurveys als Instrument vorgesehen als auch Grundlage für Indikatoren im Rahmen der europäischen Gesundheitsberichterstattung. Zudem wurde bei der Konzeption des Fragebogens darauf geachtet, möglichst viele In- dikatoren für ein europäisches GBE-System mit den Surveydaten füllen zu können [13].

Neben diesen Aspekten, die auf Vergleichbarkeit und Kontinuität zielen, konnte die Flexibilität der Interviewsurveys genutzt werden. Als der erste Telefongesundheitssurvey konzipiert wurde, konkretisier- 


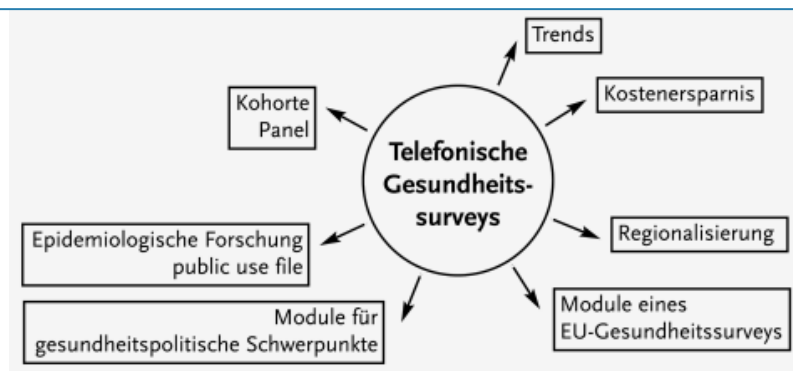

Abb. $2<$ Nutzungsmöglichkeiten der Telefonischen Gesundheitssurveys ten sich in der gleichen Zeit die Aktionsfelder des Gesundheitszielprogramms auf Bundesebene „gesundheitsziele.de“ [14]. Diese Aktionsfelder betreffen u. a. Diabetes mellitus, chronische Rückenschmerzen, gesundheitliche Kompetenz von Bürgern und Patienten, Brustkrebs sowie Ziele für das Kindes- und Jugendalter. Um die Datenbasis vor Beginn des Zielprogramms zu verbessern, wurden zu fast allen Bereichen Fragen in das Spektrum des Telefonsurveys integriert bzw. deutlich ausgebaut. Auch der Bereich Bürger- und Patientenorientierung, insbesondere Aspekte der Transparenz (Gesundheitsinformationen) und Kompetenz (Selbsthilfe), wurde im Vergleich zu den bisherigen Gesundheitssurveys verstärkt berücksichtigt. Zusätzlich enthält der Themenkanon des Telefonsurveys ein umfangreiches Modul für den Bereich chronischer Rückenschmerz sowie Diabetes mellitus: So wurden über 30 Fragen zum Themenkomplex Rückenschmerzen gestellt, die es ermöglichen, repräsentative Aussagen zur Prävalenz - speziell auch von chronischen Rückenschmerzen - zu treffen und das diesbezügliche Versorgungsgeschehen zu beschreiben. Die Inanspruchnahme medizinischer Leistungen wurde detailliert erfragt, insbesondere die durchgeführte Diagnostik und Therapie (ambulant, akut stationär und während der Rehabilitation).

Im Ergebnis umfasst das Themenspektrum des GSTelo3 die folgenden Oberbereiche, die in $\bullet$ Tabelle 1 auszugsweise weiter aufgeschlüsselt werden:

- subjektive Gesundheitswahrnehmung und gesundheitsbezogene Lebensqualität,

- Risikofaktoren,

- gesundheitsbezogenes Verhalten und Prävention,

- Krankheitsgeschehen,

- Krankheitsfolgen und Behinderung,

- Inanspruchnahme von Leistungen des Gesundheitssystems,
- Gesundheitswissen, Informationsverhalten, gesundheitliche Einstellungen, - soziale Faktoren, background variables.

Der vollständige eingesetzte Fragebogen kann über das Robert Koch-Institut (gbe@rki.de) bezogen werden.

\section{Fazit und Ausblick}

Mit dem ersten bundesweiten Telefongesundheitssurvey wurde ein weiterer Baustein eines Gesundheitsmonitorings auf Bundesebene etabliert. Die Ergebnisse dieser Erhebung wurden und werden umfangreich genutzt (• Abb. 2). So liefern sie wichtige Informationen, die in die Gesundheitsberichterstattung des Bundes einfließen und bisherige Datenlücken schließen. Auch der Bereich Gesundheit im zweiten Armut- und Reichtumsbericht der Bundesregierung greift auf die Daten dieses Surveys zurück [15].

Eine Reihe wissenschaftlicher Publikationen wurde bzw. wird zurzeit auf Basis der Daten des Telefongesundheitssurveys erarbeitet, darunter z. B. $[16,17,18,19,20$, $21,22,23]$ sowie die Beiträge in diesem und im folgenden Heft des Bundesgesundheitsblattes. Zudem werden am RKI zurzeit mehrere Forschungsvorhaben (darunter zur Prävalenz und Versorgung chronischer Rückenschmerzen, chronischer Kopfschmerzen und Schwindelerkrankungen) durchgeführt, die auf den Daten der telefonischen Gesundheitssurveys beruhen. Aktuell wird vom RKI ein Scientific-use-file einschließlich einer umfangreichen Dokumentation der Surveys vorbereitet. Damit stehen diese Daten dann auch weiteren Forschungsgruppen und Universitäten für eigene Auswertungen und Publikationen zur Verfügung.

Mit finanzieller Unterstützung des BMGS konnten weitere bundesweite Telefongesundheitssurveys in den Jahren 2004 und 2005 durchgeführt werden. Deren
Auswertung und Publikation wird gegenwärtig vorbereitet. In beiden Erhebungswellen wurden zentrale Fragebatterien weiterhin mitgeführt, um wichtige Zeitreihen fortzuführen. Zudem ergab sich die Möglichkeit, in den neuen Erhebungswellen zusätzliche Module (z. B. zum Gesundheitswissen, zu gesundheitlichen Kontrollüberzeugungen oder zur Ernährung) aufzunehmen, um den weiteren Datenbedarf, insbesondere der Gesundheitsberichterstattung, decken zu können.

\section{Längsschnittaspekte}

In Ergänzung zu dieser querschnittsorientierten Fortführung der Surveys wurden in der Welle 2005 etwa 4400 Probanden, die bereits am Telefonsurvey 2003 teilgenommen und sich dort zu einer erneuten Befragung bereit erklärt hatten, nochmals interviewt. Damit sind die Datengrundlagen für eine Längsschnittkomponente im Rahmen eines Gesundheitsmonitorings gelegt.

\section{Regionalisierung}

Die bundesweiten Gesundheitssurveys ermöglichen aufgrund ihres Stichprobendesigns repräsentative Aussagen über wichtige Gesundheitsaspekte auf Bundesebene sowie für Ost-West- oder Stadt-LandVergleiche. Für regionale Aussagen, z. B. auf Ebene der Bundesländer, ist eine Stichprobenaufstockung erforderlich, die beim Bundes-Gesundheitssurvey 98 mit dem Bayern-Modul oder beim Kinder- und Jugendgesundheitssurvey mit dem Schleswig-Holstein-Modul auch realisiert wurde. Diese Option besteht natürlich auch für die Telefonsurveys. Ihre zugrunde liegende Stichprobenziehung erlaubt es prinzipiell, auch kleinräumige Analysen durchzuführen, wobei der jetzige Stichprobenumfang jedoch differenzierte kleinräumige Analysen schnell begrenzt. Perspektivisch bieten sich auch bei den telefonischen Gesundheitssurveys regionale Aufstockungen bei der Stichprobenziehung an. Diese im Vergleich zu den Untersuchungssurveys finanziell deutlich günstigere Variante kannt regionalspezifische Informationen zur Gesundheit der jeweiligen Bevölkerung liefern, die unmittelbar mit den methodisch identisch erhobenen 
Bundesdaten abgeglichen werden können, um lokale bzw. regionale Maßnahmen bedarfsgerecht durchzuführen.

\section{Gesundheitspolitische Rolle}

Sowohl in der Selbstverwaltung als auch bei den gesundheitspolitischen Akteuren auf kommunaler, Landes- und Bundesebene wurden in den letzten Jahren Anstrengungen unternommen, um Prävention, Gesundheitsförderung und Gesundheitszieleprozesse zu stärken. Gesundheitssurveys spielen dabei in doppelter Hinsicht eine wichtige Rolle. Zum einen erlauben sie es, das Gesundheitsverhalten in verschiedenen Altersgruppen, sozialen Schichten und gesellschaftlichen Bezügen zu beschreiben und zu analysieren. Sie liefern damit die wissenschaftlich fundierte Grundlage für die Identifizierung von Personengruppen oder auch Settings, bei denen Präventions- oder Gesundheitsförderungsmaßnahmen zu einem bestmöglichen Ergebnis führen können (Identifikation von Präventionspotenzialen). Zum anderen spielen Gesundheitssurveys eine wichtige Rolle bei der Steuerung, Anpassung und Evaluation der Maßnahmen auf Bevölkerungsebene. Ihre regelmäßige Durchführung trägt dazu bei, die Ergebnisse von Programmen und Aktivitäten zu beurteilen, beispielsweise durch das Aufzeigen erfolgter oder ausbleibender Veränderungen im Gesundheitswissen, im Gesundheitsverhalten und im Idealfall auch im Gesundheitszustand der Bevölkerung. Zusätzlich zur Evaluation von Präventionsprogrammen im Sinne eines Vorher-Nachher-Vergleichs dienen Gesundheitssurveys auch der Steuerung und Anpassung dieser Programme: Daten des Telefonsurveys 2003 und seiner Nachfolger spielen z. B. eine Rolle im Evaluationskonzept des Programms gesundheitsziele.de. Hier können beispielsweise im Bereich des Gesundheitsziels „Tabakkonsum reduzieren“ intendierte Verhaltens- und Einstellungsänderungen zum Rauchen im Verlauf des Gesundheitszielprozesses mit den Daten aus regelmäßigen Gesundheitssurveys nachvollzogen werden. Durch ein zeitnahes Monitoring der gewünschten Änderungen ergibt sich die Möglichkeit, Maßnahmenprozesse ggf. zielorientiert anzupassen.

Wie sieht nun die längerfristige Perspektive der telefonischen Gesundheitssurveys aus? Es ist geplant, auch im Jahr 2006 eine neue Erhebung durchzuführen. Die Tatsache, dass es damit dann gelungen wäre, bereits 4 Wellen eines jährlichen Gesundheitssurveys zu realisieren, kann aber nicht darüber hinwegtäuschen, dass ihre dauerhafte Finanzierung noch nicht sichergestellt ist. Die bisher in unregelmäßigen $\mathrm{Ab}$ ständen durchgeführten bundesweiten $\mathrm{Ge}$ sundheitssurveys folgen zwar einem einheitlichen Konzept, jedoch werden sie bisher als eigenständige Projekte realisiert, deren jeweilige Finanzierung mit einem erheblichen Aufwand über einzuwerbende Drittmittel sichergestellt werden muss. Für regelmäßige Gesundheitssurveys, die sowohl der epidemiologischen Public-Health-Wissenschaft, der Gesundheitsberichterstattung als auch der Planung von evidenzbasierten Präventionsmaßnahmen dienen, ist eine dauerhafte Finanzierung aber unabdingbare Voraussetzung.

\section{Korrespondierender Autor \\ Dr. T.Ziese}

Robert Koch-Institut

Postfach 650261, 13302 Berlin

E-Mail: zieset@rki.de

\section{Literatur}

1. Kurth B, Ziese T, Tiemann F (2005) Gesundheitsmonitoring auf Bundesebene: Ansätze und Perspektiven. Bundesgesundheitsblatt Gesundheitsforschung Gesundheitsschutz 48:261-272

2. Robert Koch-Institut (2004) Telefonischer Gesundheitssurvey des Robert Koch-Instituts zu chronischen Krankheiten und ihren Bedingungen. Beiträge zur Gesundheitsberichterstattung des Bundes. Eigenverlag, Berlin

3. Land $\mathrm{G}$ (2002) Measuring 2010 national objectives and leading indicators at the state and local level. $J$ Public Health Manag Pract 8(4):9-13

4. Aromaa A, Koponen P, Tafforeau J et al. (2003) Status and future of health surveys in the European Union. Final report of Phase 2 of the project health surveys in the EU: HIS and HIS/HES evaluations and models. Publications of National Public Health Institute 182

5. Meyer N, Fischer R, Weitkunat R et al. (2002) Evaluation of health monitoring in Bavaria by computer-assisted telephone interviews (CATI) in comparison to the German National Health Examination Survey conducted in 1998 by the Robert Koch Institute. Gesundheitswesen 64(6):329-336

6. Bradburn N (1999) Discussion of papers on mode effects. In: Cynamon M, Kulka R (eds) Seventh conference on health survey research methods. Department of Health and Human Services, Centers for Disease Control and Prevention, National Center for Health Statistics Williamsburg, Virginia, pp 155-157
7. de Leeuw E, van der Zouwen J (1988) Data quality in telephone and face to face surveys: a comparative metaanalysis. In: Groves R, Biemer P, Lyberg L (eds) Telephone survey methodology. John Wiley \& Sons, New York, pp 283-299

8. Green M, Krosnick J (1999) Comparing telephone and face-to-face interviewing in terms of data quality: the 1982 National Election Studies Method Comparison Project. In: Cynamon M, Kulka R (eds) Seventh conference on health survey research methods; 1999 February 2001. Department of Health and Human Services, Centers for Disease Control and Prevention, National Center for Health Statistics Williamsburg, Virginia, pp 115-121

9. Nelson D, Holtzman D, Bolen J et al. (2001) Reliability and validity of measures from the Behavioral Risk Factor Surveillance System (BRFSS). Soz Praventivmed 46 [Suppl 1)]:3-42

10. Nelson D, Powell-Griner E, Town M et al. (2003) A comparison of national estimates from the National Health Interview Survey and the Behavioral Risk Factor Surveillance System. Am J Public Health 93(8):1335-1341

11. Mokdad A, Serdula M, Dietz W et al. (1999) The spread of the obesity epidemic in the United States, 1991-1998. JAMA 282(16):1519-1522

12. Sanderson D, Nørlev J, Iburg K et al. (2002) Percieved health. In: Robine J, Jagger C, Romieu I (eds) Selection of a coherent set of health indicators for the European Union. Euro-Reves Montpellier, pp 81-95

13. Kramers $P$ (2003) The ECHI project: health indicators for the European Community. Eur J Public Health 13 [Suppl 1]

14. www.gesundheitsziele.de (2005) Gemeinsames Vorhaben des Bundesministeriums für Gesundheit und Soziale Sicherung und der Gesellschaft für Versicherungswissenschaft und -gestaltung e.V. (GVG) www.gesundheitsziele.de (25.7.2005)

15. Bundesministerium für Gesundheit und Soziale Sicherung (2005) Lebenslagen in Deutschland - Der 2. Armuts- und Reichtumsbericht der Bundesregierung. Berlin, Februar 2005, http://www.bmgs. bund.de/deu/gra/publikationen/publ_ausgabe. php?gruppe $=19$ \&lang $=$ deu

16. Ellert U, Lampert T, Neuhauser $\mathrm{H}$ et al. (2004) Health related quality of life measured with the SF-8: results of the German National Health Telephone Survey 2002/2003. J Epidemiol Community Health 58:A79

17. Lampert T, Burger M (2004) Rauchgewohnheiten in Deutschland - Ergebnisse des telefonischen Bundes-Gesundheitssurveys. Gesundheitswesen 66:511-517

18. Neuhauser H, Ellert U, Ziese T (2004) Chronische Rückenschmerzen in der Allgemeinbevölkerung in Deutschland: Prävalenz und Risikogruppen. (eingereicht)

19. Neuhauser $H$, Radtke A, von Brevern M et al. (2004) Migrainous vertigo in the general population in Germany. Akt Neurologie 31:\$63

20. Neuhauser H, Radtke A, von Brevern M et al. (2004) Prevalence of vestibular vertigo in Germany: a population-based study. J Neurology 251 [Suppl 3]:170-171

21. Neuhauser H, Ziese T (2004) Chronic back pain in the general population in Germany. Results from the National Telephone Health Survey 2002/2003. Biometrical J 46 [Suppl 1]:118

22. Radtke A, Neuhauser $H$, von Brevern $M$ et al. (2004) Clinical consequences of orthostatic dizziness in the general population. Akt Neurologie 31:S64

23. Schenk $L$ (2004) Beteiligung von Migranten im Telefonischen Gesundheitssurvey: Möglichkeiten und Grenzen. (eingereicht) 